\title{
Effect of Ag doping on hydrothermally grown ZnO thin-film electronic synapse device
}

Navaj B. Mullani MSC

Research student, Computational Electronics and Nanoscience Research Laboratory, School of Nanoscience and Biotechnology, Shivaji University, Kolhapur, India

Vijay B. Patil MSc

Research student, Computational Electronics and Nanoscience Research Laboratory, School of Nanoscience and Biotechnology, Shivaji University, Kolhapur, India

Rahul S. Tikke MSC

Research student, Computational Electronics and Nanoscience Research Laboratory, School of Nanoscience and Biotechnology, Shivaji University, Kolhapur, India
Pravin S. Pawar MSc

Research student, Computational Electronics and Nanoscience Research Laboratory, School of Nanoscience and Biotechnology, Shivaji University, Kolhapur, India

Santosh V. Mohite PhD

Research Associate, Department of Physics, Shivaji University, Kolhapur, India Amit A. Bagade PhD

Research Associate, Department of Physics, Shivaji University, Kolhapur, India Tukaram D. Dongale PhD

Assistant Professor, Computational Electronics and Nanoscience Research Laboratory, School of Nanoscience and Biotechnology, Shivaji University, Kolhapur, India (corresponding author: tdd.snst@unishivaji.ac.in)

The present paper reports the effect of silver $(\mathrm{Ag})$ doping on hydrothermally grown zinc oxide ( $\mathrm{ZnO}$ ) thin-film memristor-based electronic synapse devices. The morphological, structural and electrical characterizations of the zinc oxide memristor devices were carried out using scanning electron microscopy, $\mathrm{X}$-ray diffraction and a programmable electrochemical workstation, respectively. The crystallographic orientation was detected along the (002) plane with well-aligned and compact zinc oxide nanorods for silver-doped zinc oxide memristor devices. An analog memory with a synaptic weight behavior similar to that of a biological synapse was observed for the developed devices. The morphological, structural, electrical and resistive switching mechanism studies clearly show improved performance of silver-doped zinc oxide devices over that of the bare zinc oxide device. Furthermore, the conduction mechanism of the devices follows the ohmic and space-charge-limited conduction theories. The present report is useful for the development and optimization of zinc oxide-based electronic synaptic devices for neuromorphic applications.

\section{Notation \\ $D \quad$ crystallite size of the sample \\ $\beta \quad$ full width at half maximum \\ $\theta \quad$ Bragg's angle \\ $\lambda \quad$ wavelength of the $\mathrm{X}$-rays}

\section{Introduction}

Neuromorphic computing is speculated as the base of future artificial intelligence technology. In order to develop highly scalable and efficient neuromorphic computing architecture, it is a need of the hour to develop a fundamental building block that can mimic the various properties of biological neurons and synapses. ${ }^{1}$ Among the many alternatives for neuromorphic computing, the memristor was widely studied due to its non-volatility, data retention, non-linearity, two-terminal structure and passivity property. ${ }^{2-6}$ Along with these, the compatibility of the simple metal-insulator-metal (MIM) structure of a memristor with the conventional complementary metal-oxide-semiconductor fabrication process offers an advantage over other memory devices. ${ }^{7}$ In view of this, various transition metal oxide memristor devices have been developed in recent years. ${ }^{8-14}$ Among various metal oxides, zinc oxide $(\mathrm{ZnO})$ is one of the promising transition metal oxide materials for the memristor and neuromorphic applications. $^{9,12}$
The conduction mechanism of metal oxide memristor devices is due to the formation and breaking of conducting filaments. ${ }^{15}$ Zinc oxide is a semiconducting insulating material that has a fewer number of ions/oxygen vacancies. A high operating voltage and a slow switching speed are inadequate for the utilization of zinc oxide for resistive memory and neuromorphic applications. Change in the concentration of the ions/oxygen vacancies facilitates better resistive switching property. Dopants are used for enhancing the electrical, optical and structural properties of zinc oxide thin films. ${ }^{16}$ Recently, Wang et al. ${ }^{17}$ reported the resistive switching properties of a manganese (Mn)-doped zinc oxide memristor device and showed that the bipolar resistive switching was induced due to the switching of magnetic moments. Younis et al. ${ }^{18}$ studied the bistable resistive switching properties of titanium (Ti)-doped zinc oxide thin films. The reported memristor device shows enhancement in resistive switching property in the context of the high-resistance state (HRS) to lowresistance state (LRS) ratio compared to a pure zinc oxide memristor device. ${ }^{18}$ Huang et al. ${ }^{19}$ reported the highly oriented and dense compact structure of a gallium (Ga)-doped zinc oxide device. The developed device shows good HRS/LRS ratio and memory performance. ${ }^{19}$ Lee et al. ${ }^{20}$ investigated the resistive switching property of an aluminum (Al)-doped zinc oxide thin film. This work suggested that the ratio of HRS/LRS can be improved with appropriate aluminum doping. ${ }^{20}$ Among many doping elements, 
silver (Ag) is an appropriate choice due to its stability, costeffectiveness and non-toxic nature. Furthermore, silver possesses many important characteristics such as high solubility, larger ionic size and minimum orbital energy. ${ }^{21}$ Silver doping increases the distribution of surface charges, which results in better electrical and optical properties. ${ }^{22}$ Considering the importance of silver doping and literature trend, it is important to investigate the effect of silver doping on zinc oxide thin-film memristor devices. In the present investigation, the authors have altered the silver doping concentration and studied the corresponding effects on structural properties, morphological properties, analog memory, synaptic weights, resistive switching voltages and conduction mechanism of zinc oxide memristor devices.

The structural and morphological properties were investigated using X-ray diffraction (XRD) and scanning electron microscopy (SEM). Furthermore, the electrical properties such as analog memory, synaptic weights and resistive switching voltages were investigated using a programmable electrochemical workstation. The rest of the paper is organized as follows: Section 1 deals with the introduction and general overview of the research problem. Following this section, Section 2 discusses the experimental details of the research work. In Section 3, the structural, morphological and electrical characterizations of memristor devices are discussed, and the conclusion has been placed at the end of the paper.

\section{Experimental details}

\subsection{Materials and methods}

In the present study, silver-doped aluminum/zinc oxide/SS MIM devices were fabricated using a simple and low-cost hydrothermal method. Aluminum and stainless steel (SS) were used as the top and bottom electrodes, respectively. Zinc oxide was used as an active layer for the memristor device. The single-step and solution-phase-based low-temperature hydrothermal route was used to fabricate the devices. A simple laboratory autoclave was used for the deposition process. Zinc acetate $\left(\mathrm{Zn}\left(\mathrm{O}_{2} \mathrm{CCH}_{3}\right)_{2} ; 99 \%\right.$, Sigma-Aldrich) and extra pure silver nitrate $\left(\mathrm{AgNO}_{3}\right.$; SigmaAldrich) were used as source materials. Water was used as the solvent during the entire process, and ammonia $\left(\mathrm{NH}_{3}\right)$ solution (about 30\%) was used for the complex formation and to act as the reducing agent. All the chemicals and reagents were of analytical grade and used as received without any further treatments.

\subsection{Deposition of zinc oxide and silver-doped zinc oxide thin films}

Bare zinc oxide and silver-doped zinc oxide thin films were deposited on 304 grade SS substrate by a hydrothermal technique using zinc acetate and silver nitrate as source materials. All the glassware was washed properly before beginning the procedure and rinsed with double-distilled water (DDW). One side of the SS substrate was polished with zero-grade polish paper to remove the roughness of the substrate. The precursor concentration was optimized at $0.09 \mathrm{M}$ in order to synthesize the nanostructured zinc oxide thin films. First, $0.09 \mathrm{M}$ of zinc acetate was dissolved in $50 \mathrm{ml}$
DDW under continuous stirring. The ammonia solution was added dropwise in the mentioned solution with vigorous stirring until the solution became colorless. The polished SS substrate $(4 \mathrm{~cm} \times 1 \mathrm{~cm})$ was inserted into the solution, and the beaker was partially closed with aluminum foil. The solution was kept for the deposition in an autoclave at $90^{\circ} \mathrm{C}$ for $1 \mathrm{~h}$. The deposited substrate was removed and rinsed with DDW and kept for air-drying. This sample was labeled A1. To begin the synthesis of silver-doped zinc oxide thin films, the concentration of zinc acetate was fixed at $0.09 \mathrm{M}$, while the silver nitrate had various molar percentages $(1-3 \%)$. In order to develop $1 \mathrm{~mol} \%$ silver-doped zinc oxide thin film, solutions of optimized concentrations were prepared, and from these, $49.5 \mathrm{ml}$ of zinc acetate and $0.5 \mathrm{ml}$ of silver nitrate were added together in a beaker and the total volume of the solution was made equal to $50 \mathrm{ml}$. The solutioncontaining beaker was kept for continuous stirring on a magnetic stirrer for $5-10 \mathrm{~min}$. The ammonia solution was added dropwise in the mentioned solution with dynamic stirring until the solution became colorless. The polished SS substrate $(4 \mathrm{~cm} \times 1 \mathrm{~cm})$ was inserted into the solution, and the beaker was partially closed with aluminum foil. The reaction time and parameters were kept similar to those for A1. The deposited substrate was removed and rinsed with DDW and kept for air-drying. This sample was labeled B2. In order to investigate the effect of silver doping on the zinc oxide memristor device, 2 and 3\% silver-doped zinc oxide thin films were prepared by keeping the whole procedure and parameters similar to those for B2. These samples were labeled B3 and B4, respectively.

\subsection{Characterizations of silver-doped zinc oxide thin films}

A Bruker X-ray diffractometer model D2 phaser with copper $(\mathrm{Cu})$ $\mathrm{K} \alpha$ radiation $(\lambda=1.5406 \AA)$ was used to study the phase purity and crystal structure of the developed thin films. SEM with a Jeol JSM-6360 A microscope was used to examine the surface morphology of the active layer. The electrical measurements were carried out a using programmable electrochemical workstation (Autolab $\mathrm{N}$ series) controlled with the Nova software. The top electrode aluminum was biased, whereas the bottom SS electrode was grounded during all electrical measurements.

\section{Results and discussion}

\subsection{Morphological and structural characterization of zinc oxide active layers}

XRD characterization was used to investigate the crystallinity of the zinc oxide active layer. Curves a-d in Figure 1 are the XRD patterns of bare and silver-doped zinc oxide thin-film memristor devices. The obtained XRD patterns are well matched with JCPDS card number 01-079-2205, which shows a polycrystalline hexagonal wurtzite structure with a preferred (002) orientation. The appearance of the * peak represents the SS substrate. The intensity of the (002) plane increases as the amount of the dopant material increases. The significant decrease in the (002) and (101) planes that are observed in the B4 device may be due to the doping-induced stress in the lattice, ${ }^{23}$ as shown by curve $d$ in Figure 1 . The crystallite size was calculated from Scherrer's formula as given below 
Bioinspired, Biomimetic and Nanobiomaterials Volume 7 Issue BBN2
Effect of Ag doping on hydrothermally grown $\mathrm{ZnO}$ thin-film electronic synapse device

Mullani, Patil, Tikke et al.

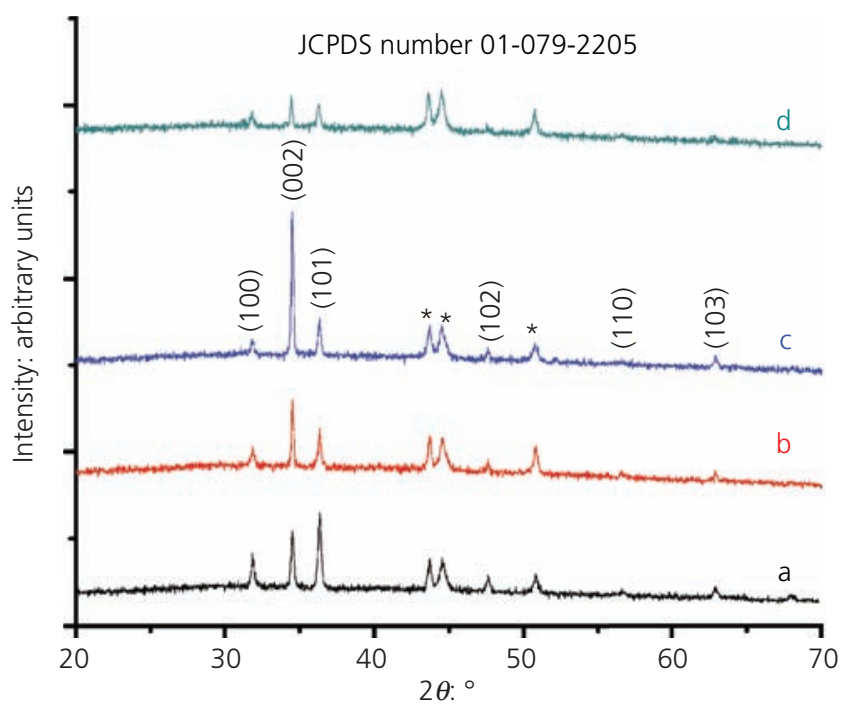

Figure 1. XRD patterns of the bare zinc oxide (curve a) and silverdoped zinc oxide active layer with doping varied from 1 to $3 \%$ (curves b-d). JCPDS, Joint Committee on Powder Diffraction Standards

1. $D=\frac{0 \cdot 9 \lambda}{\beta \cos \theta}$ where $D$ is the crystallite size of the sample, $\lambda$ is the wavelength of the X-rays, $\beta$ is the full width at half maximum and $\theta$ is the Bragg angle. The crystallite size was calculated along the (002) plane, and it was found to be $70 \mathrm{~nm}$ (A1), $53 \mathrm{~nm}$ (B2), $60 \mathrm{~nm}$ (B3) and $54 \mathrm{~nm}$ (B4). This confirms that the doping of silver in the zinc oxide active layer leads to a decrease in crystallite size compared to bare zinc oxide. $^{24,25}$ Figures 2(a)-2(d) show the SEM micrograph of bare and silver-doped zinc oxide thin-film memristor devices. A non-aligned and irregular zinc oxide nanorod morphology was observed for the bare zinc oxide thin-film memristor device (A1). The subsequent SEM images in Figures 2(b)-2(d) reveal a change in the morphology of the zinc oxide active layer due to the increase in the silver doping. Well-aligned, thin nanorods with narrow rod-spacing-like morphology were observed for the $\mathrm{B} 2, \mathrm{~B} 3$ and $\mathrm{B} 4$ devices compared to the $\mathrm{A} 1$ device. Furthermore, the $\mathrm{B} 2$ and $\mathrm{B} 4$ devices show random formation of silver stacks or agglomeration, whereas the B3 device indicates uniform and homogenous distribution of silver. This is due to fact that the crystallite size of the B3 sample is higher than those of the B2 and B4 samples. It is a well-known fact that lower-crystallite-size materials possess a higher surface energy, and in order to reduce the surface energy and to attain stability or the equilibrium state, agglomeration takes place. On the other hand, higher-crystallite-size materials have a sufficiently lower surface energy, which can prevent agglomeration. The results clearly indicate that silver doping assists to control the uniform distribution and enhances the overall alignment orientation of zinc oxide nanorods. ${ }^{26}$

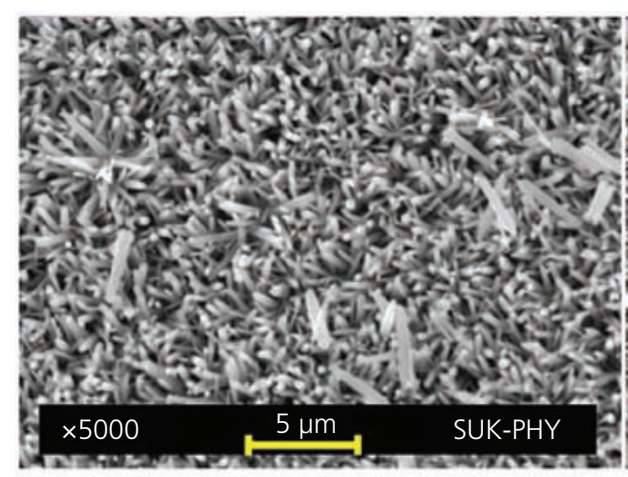

(a)

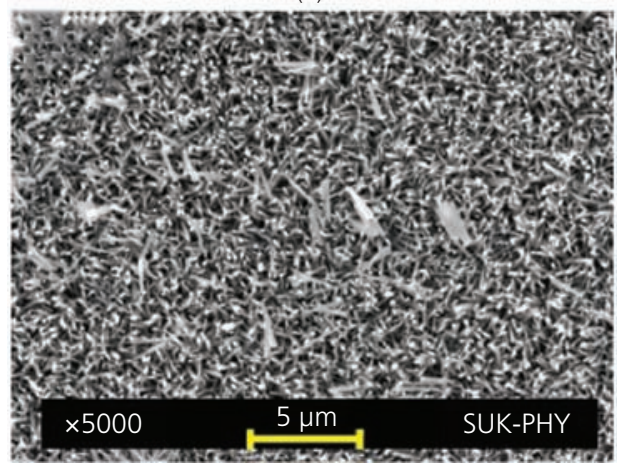

(c)

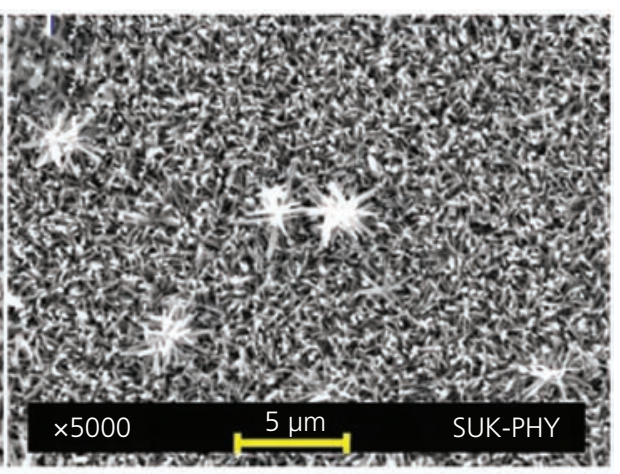

(b)

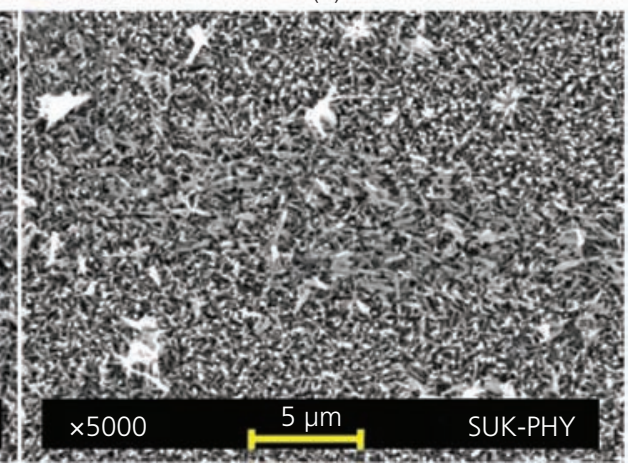

(d)

Figure 2. SEM images of the (a) zinc oxide and (b-d) silver-doped zinc oxide active layer with doping varied from 1 to $3 \%$ 
Bioinspired, Biomimetic and Nanobiomaterials Volume 7 Issue BBN2
Effect of Ag doping on hydrothermally grown $\mathrm{ZnO}$ thin-film electronic synapse device

Mullani, Patil, Tikke et al.

\subsection{Analog memory and synaptic weight behavior of aluminum/zinc oxide/SS thin-film memristor}

The analog memory property was confirmed by altering the sweep rates of the silver-doped zinc oxide memristor devices. Analog memory and synaptic weight behavior are some of the essential properties that have to be emulated by a memristor in order to work as an electronic synapse. In a biological synapse, chemical or electrical signals are responsible for the fusing of neurotransmitters in the synaptic cleft for altering the synaptic weights. The current $(i(t))$ flowing through the memristor and its magnitude are analogous with the neurotransmitters and synaptic weights of a biological synapsee. ${ }^{2,13,14}$ In the present case, all devices exhibit pinched hysteresis loop property on $I-V$ plane. Figures $3(\mathrm{a})-3(\mathrm{c})$ clearly evidence that the developed devices show analog memory with asymmetric bipolar resistive switching property. The term 'analog' indicates the smooth increase or decrease in the current $(i(t))$. In general, a bidirectional signal flow is observed in biological neurons and synapses ${ }^{27}$ hence, the bipolar resistive switching mechanism is advantageous for mimicking the signal flow of neurons and synapses. In addition to this, the two-terminal structure of the developed devices rightly mimics the two-terminal structure of biological neurons and synapses. The results of analog memory, bipolar resistive switching and two-terminal structure put forth the developed memristor device as a suitable candidate for synaptic device development.

The synaptic weight behaviors at the positive- and negative-bias regions with different sweep rates are shown in Figures 4(a) and 4(b). The results clearly show the silver-doping-dependent synaptic weight behavior of zinc oxide thin-film memristor. The results reveal that the A1 device shows a slight increase in synaptic weights at the positive-bias region, while it shows a decrease in the negative-bias region. In comparison with the A1 device, the subsequent devices show a decrease in synaptic weights in the positive-bias region and an increase in the negative-bias region. Higher synaptic weights were observed for B3 devices in both regions due to the well-aligned nanorod structure and uniform silver doping. On the other hand, the synaptic weights of the $\mathrm{B} 2$ and $\mathrm{B} 4$ devices decrease due to the

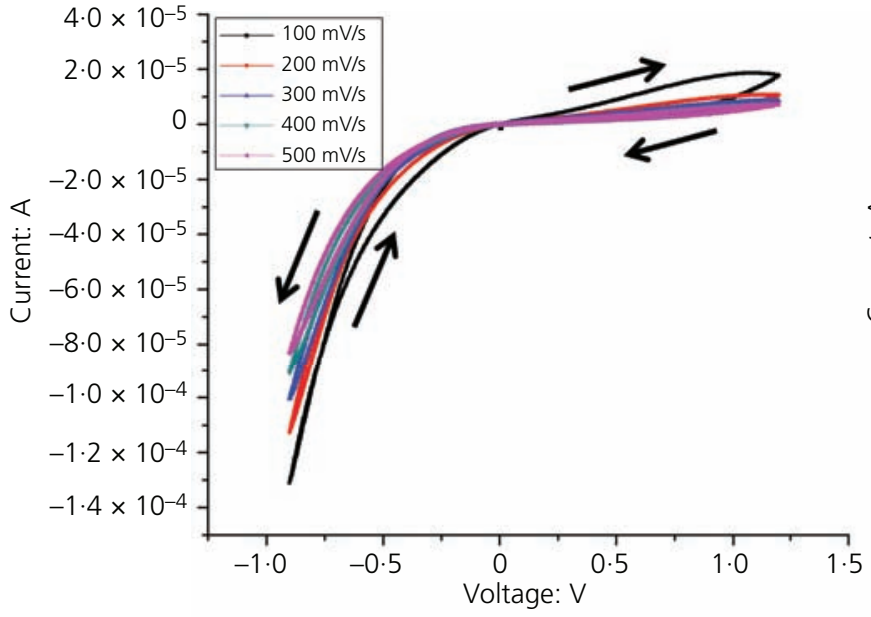

(a)

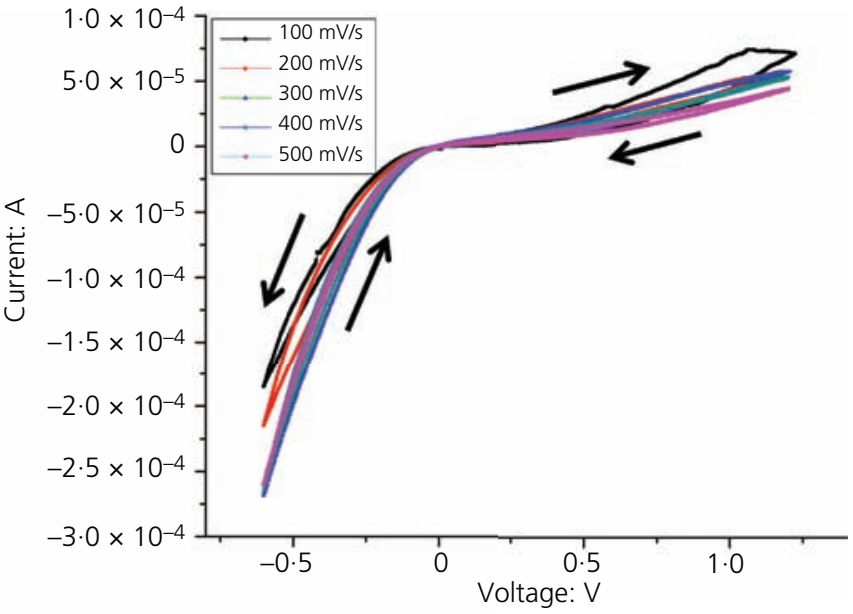

(b)

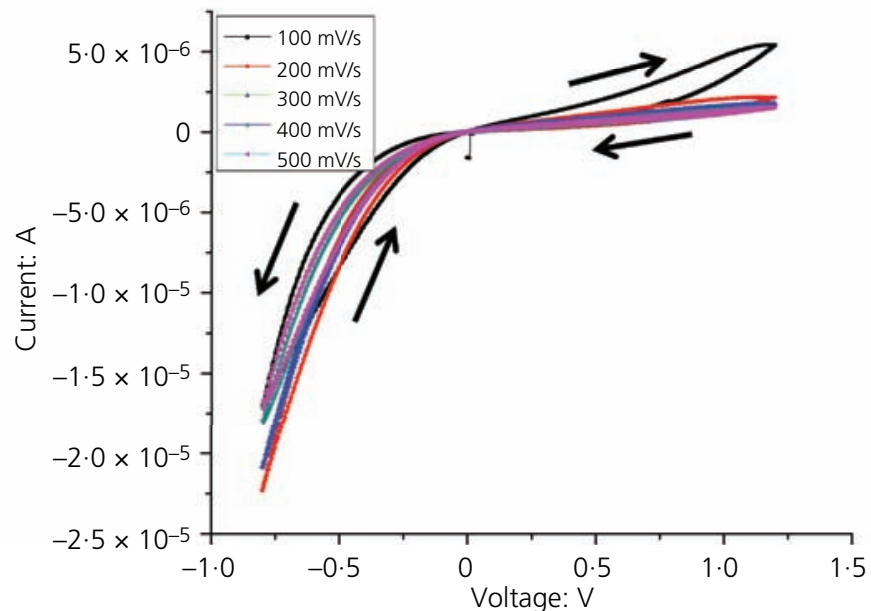

(c)

Figure 3. Analog memory property of silver-doped aluminum/zinc oxide/SS thin-film memristor devices measured at different sweep rates $(100-500 \mathrm{mV} / \mathrm{s})$ for doping concentrations of (a) 1, (b) 2 and (c) 3\%. The arrows indicate the direction of resistive switching 


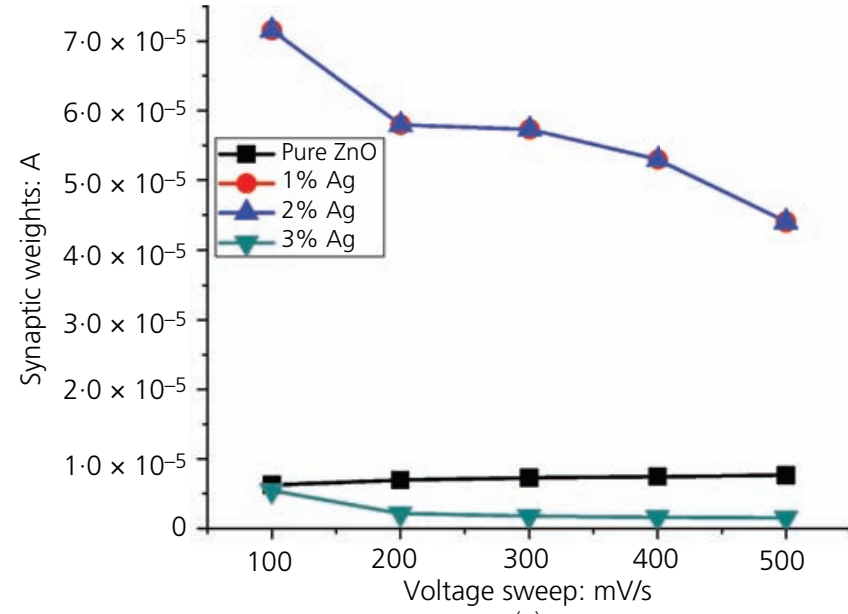

(a)

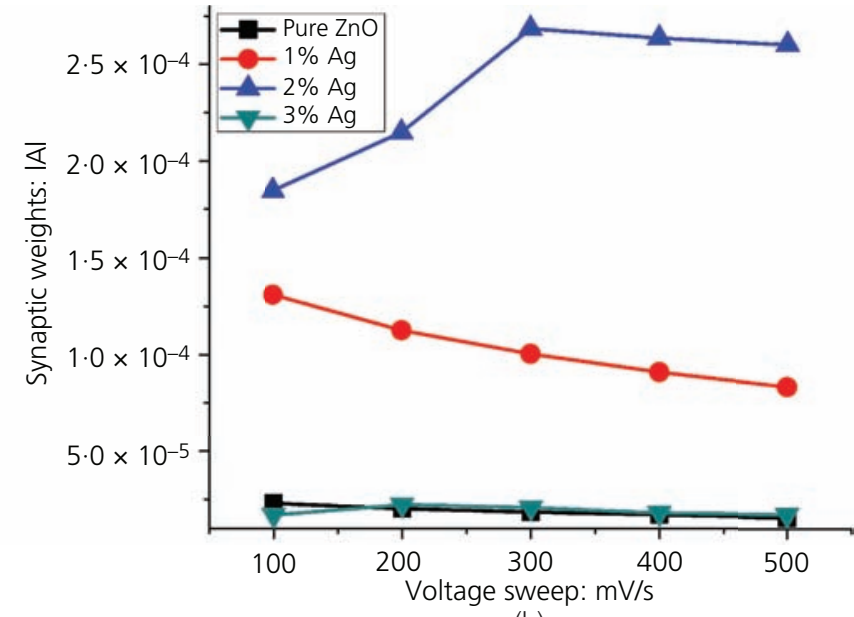

(b)

Figure 4. Synaptic weight behaviors of aluminum/zinc oxide/SS and aluminum/silver-zinc oxide/SS thin-film memristor devices. (a) Synaptic weights at positive bias with different sweep rates; (b) synaptic weights at negative bias with different sweep rates

increase in the leakage current. The increase in the leakage current is due to the highly non-uniform silver doping and grain boundary effect. ${ }^{13,14}$ Furthermore, it is interesting to note that the developed B3 device shows a quasi-reverse rectifying behavior. This type of device is generally used for unidirectional data flow. A unidirectional data flow enhances the computational capabilities of the neurons and synapses., ${ }^{2,28}$ This type of unique property will be useful for enhancing the computational capabilities of neuromorphic architecture by employing a unidirectional electronic synapse.

\subsection{Effect of silver doping on resistive switching voltage of zinc oxide memristor}

In order to mimic biological synaptic properties, the primitive building block of neuromorphic architecture must work on a low operating voltage. A memristor is an inherently passive device. ${ }^{29}$ The passivity arises due to the zero crossing property, in which the magnitude of the current becomes zero at zero voltage. In view of this, a resistive switching memristor is an ideal candidate for the development of synaptic devices. In the present investigation, the authors studied the effect of silver doping on the resistive switching voltages of zinc oxide memristor devices, as shown in Figure 5. An asymmetric resistive switching voltage nature was observed for the developed memristor devices. Furthermore, lower resistive switching voltages ( $V_{\text {set }}$ and $\left.V_{\text {reset }}\right)$ were observed for all developed devices. It is interesting to note that the silver-doped zinc oxide thin films show lower bipolar resistive switching voltages as compared to previously reported manganese-,${ }^{17}$ titanium-,${ }^{18}$ gallium- ${ }^{19}$ and aluminum-doped ${ }^{20}$ zinc oxide thin films.

It is observed that the magnitudes of $V_{\text {set }}$ and $V_{\text {reset }}$ increase with an increase in silver doping. This may be due to the formation of higher grain boundaries with an increase in silver doping which opposes to form a complete conductive filament. In order to form a complete conductive filament or to turn on the device, a higher resistive switching voltage is required at a higher doping level. ${ }^{30}$ The formation and rupture of conducting filaments are responsible for the resistive switching mechanism. In view of this, the wellaligned and vertical nanorods provide good conducting paths for the formation of conductive filaments. The stochastic nature of formation and breaking of conductive filaments is responsible for the different magnitudes of $V_{\text {set }}$ and $V_{\text {reset }}{ }^{31}$ It is observed that the B3 device shows improved $V_{\text {reset }}$ values compared to the B2 and B4 devices. The improved resistive switching is due to the homogenous distribution of silver dopant in the zinc oxide active layer with well-aligned and vertical nanorods.

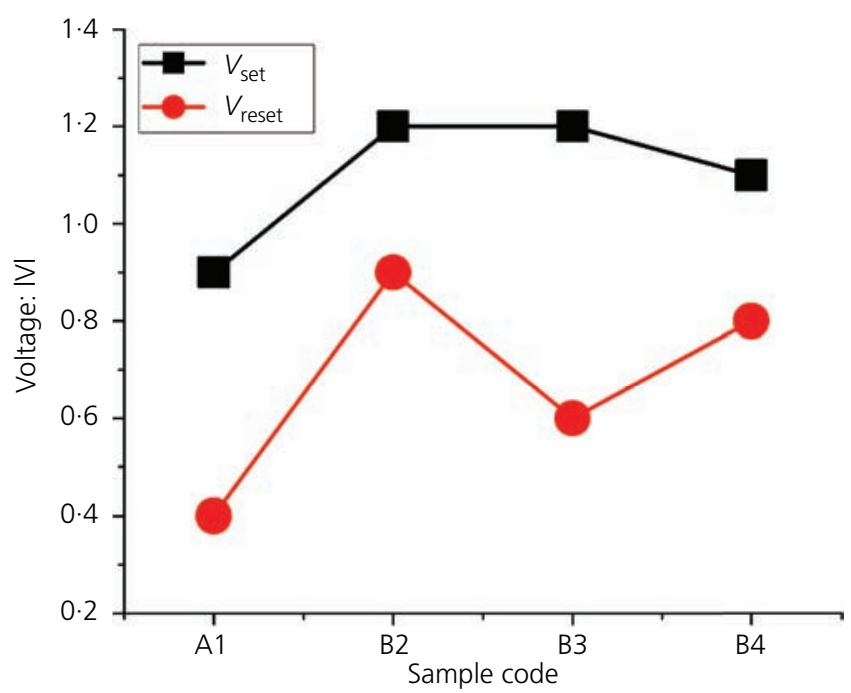

Figure 5. Effect of silver doping on resistive switching voltages ( $V_{\text {set }}$ and $V_{\text {reset }}$ ) of aluminum/zinc oxide/SS (A1) and aluminum/ silver-zinc oxide/SS (B2, B3 and B4) thin-film memristor devices 
Bioinspired, Biomimetic and Nanobiomaterials Volume 7 Issue BBN2
Effect of Ag doping on hydrothermally grown $\mathrm{ZnO}$ thin-film electronic synapse device

Mullani, Patil, Tikke et al.

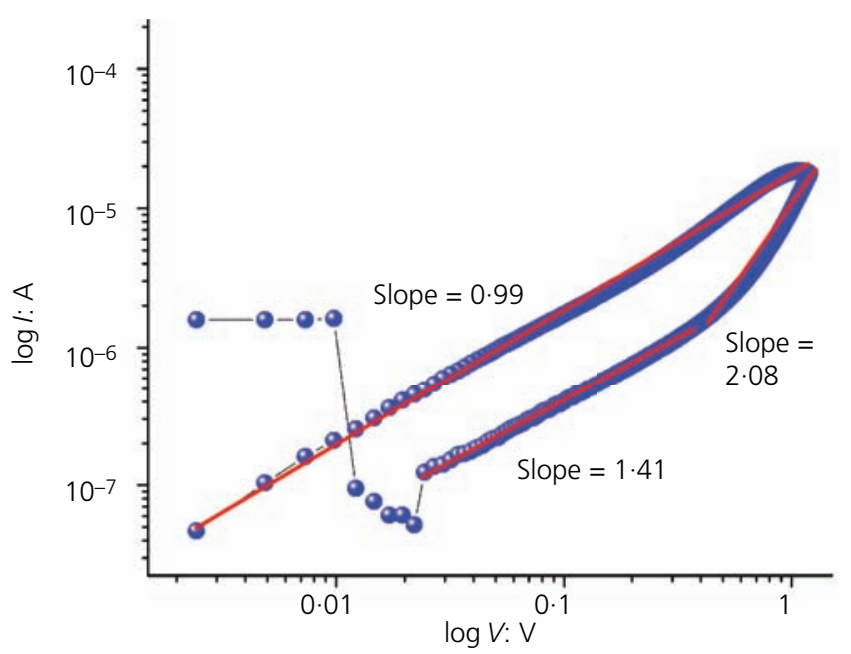

(a)

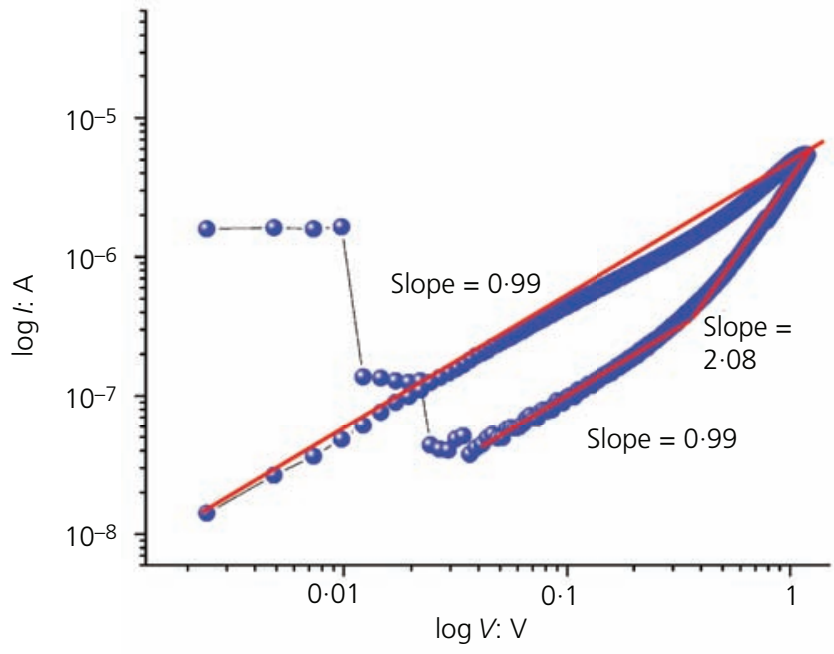

(c)

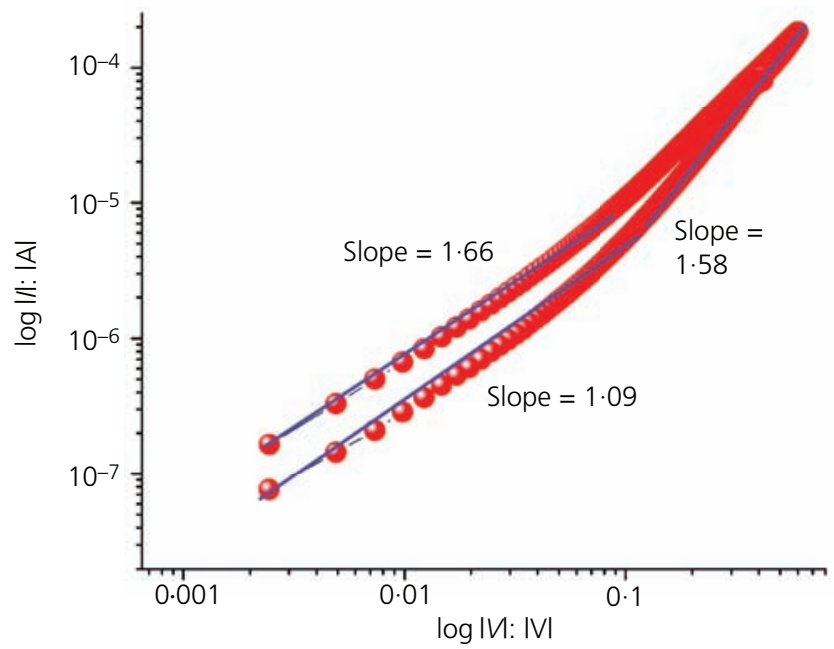

(e)

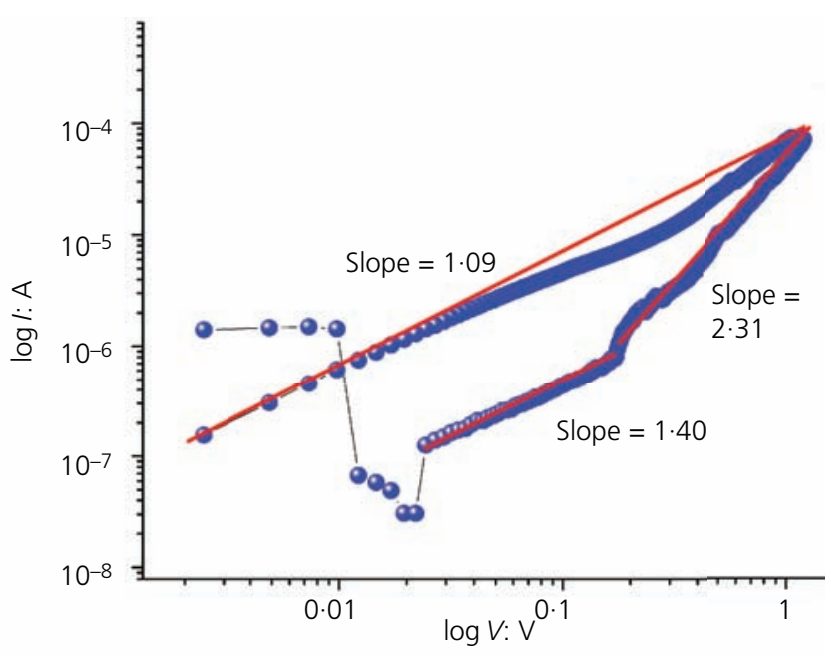

(b)

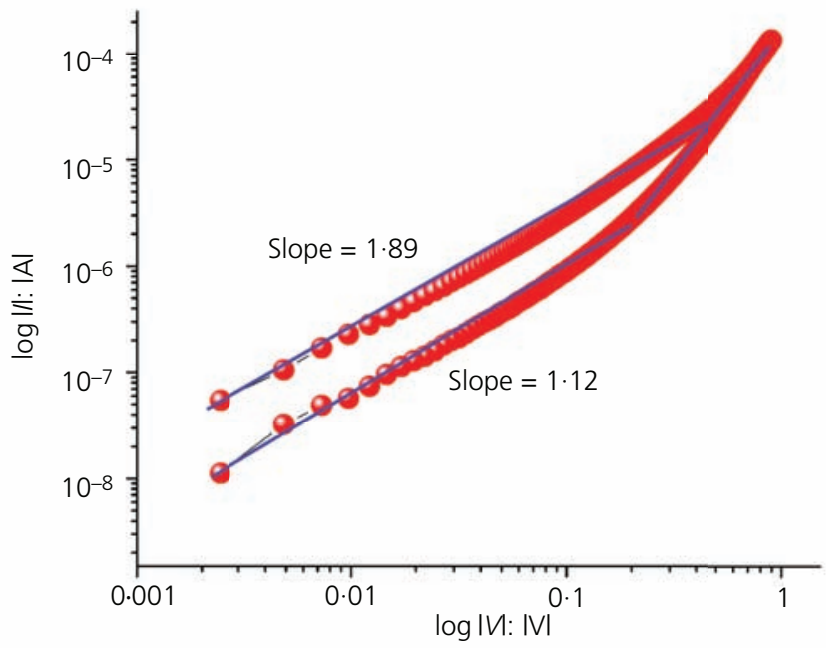

(d)

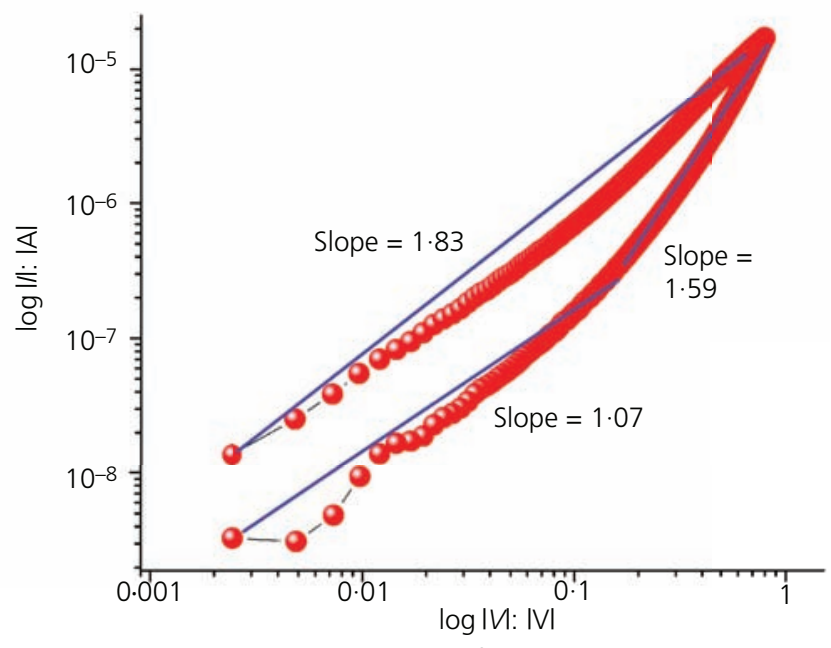

$(f)$

Figure 6. Double-logarithmic plots of current against voltage characteristics of the B2, B3 and B4 devices (a-c) at the positive-bias region and $(d-f)$ at the negative-bias region 


\subsection{Conduction mechanism and bipolar resistive switching of aluminum/zinc oxide/SS thin-film memristor}

The investigation of the conduction mechanism was carried out by plotting an $I-V$ graph on the log-log scale as shown in Figures 6(a)-(f) for the positive-bias and negative-bias regions. From the fitting of the $I-V$ graphs, it was revealed that the set state has a slope equal to 1 , which indicates that ohmic conduction dominates in this state. Following this, an abrupt increase in current was observed during the on state. The slope of this region was found to be more than 1 . This kind of data is well matched with the space-charge-limited conduction (SCLC) mechanism. ${ }^{4}$ In the negative-bias region, the reset state has a slope equal to 1 ; thus, ohmic conduction mechanism dominates in this state. In the off state, the value of the slope was found to be greater than 1; hence, SCLC is responsible for conduction in this state. In a nutshell, the ohmic and SCLC conduction mechanisms are responsible for the silver-doped zinc oxide memristor device dynamics.

The conducting filament model is generally used to describe the resistive switching mechanism in thin-film memristors. ${ }^{32}$ The conducting filament formation takes place at the defective sites of the semiconducting metal oxide layer. The constituents of the conducting filament are metal ions and oxygen vacancies present in the thin film. A schematic diagram of the bipolar resistive switching mechanism is shown in Figure 7. The resistive switching mechanism starts with the set process consisting initiation of the conducting filament composed of the active metal ions of silver in the zinc oxide active layer, as shown by point a in Figure 7. The formation of conducting filaments takes place through electrochemical metallization (ECM). As soon as a positive voltage was applied to the top electrode, reduced silver ions gather at the anode surface and conducting filament growth

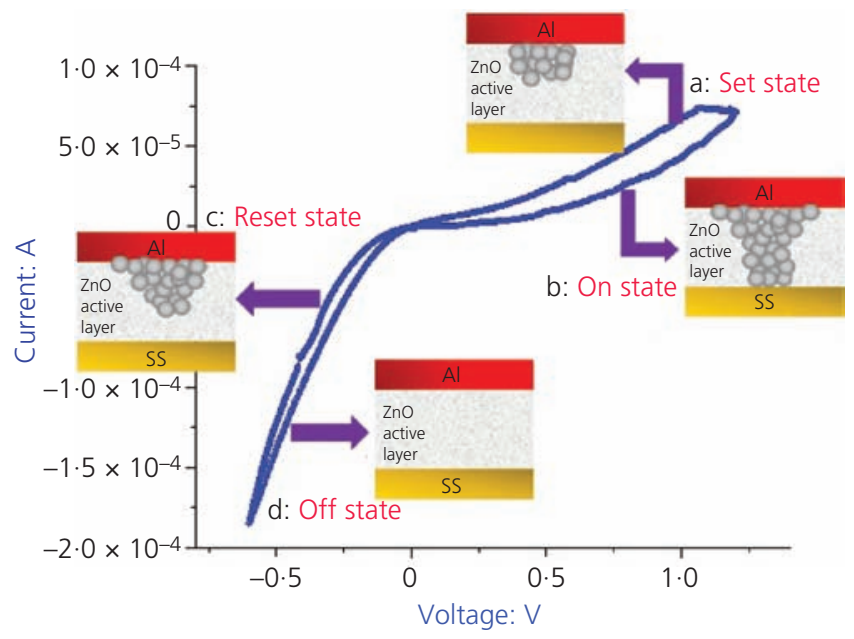

Figure 7. Possible bipolar resistive switching mechanism of silverdoped aluminum/zinc oxide/SS memristor devices. a, formation of conductive filament - that is, set state; $b$, on state of the device or LRS; $c$, breaking of conductive filament - that is, reset state; $d$, off state of the device or HRS continues until the termination at the cathode. The current conduction takes place through this conducting filament. This region of the device was considered as the on state and the corresponding voltage was termed as $V_{\text {set, }}$ as shown by point $\mathrm{b}$. The change in voltage polarity ruptures the conducting filaments, resulting in the breakdown of the current flow, and the process was known as the off state, while the corresponding voltage was termed as $V_{\text {reset, }}$ as shown by points c and d, respectively. In a nutshell, the formation and rupture of the conducting filament(s) are responsible for the bipolar resistive switching mechanism of the silver-doped zinc oxide memristor devices.

\section{Conclusion}

In the present work, the authors have investigated the effect of silver doping on the structural, morphological and electrical properties of zinc oxide thin-film memristor devices. The structural study reveals the polycrystalline nature of the active layer with the growth of nanorods along the (002) plane. Furthermore, the incorporation of silver dopant in zinc oxide thin film has produced well-aligned, highly dense and compact nanorods compared to bare zinc oxide memristor devices. The $I-V$ characteristics reveal that the developed devices show the fingerprint characteristic of a memristor and analog memory property. Furthermore, the developed devices show a silverdopant-dependent synaptic weight behavior. The developed devices possess asymmetric resistive switching property, and the magnitude of $V_{\text {set }}$ was partially dependent on the dopant concentration. The bipolar resistive switching mechanism was due to the ECM mechanism, whereas the conduction mechanism of the devices followed the ohmic and SCLC theories. In a nutshell, the $2 \%$ silver-doped zinc oxide memristor device appears as the best device compared to bare and other doped zinc oxide memristor devices due to its lower surface energy, lower resistive switching voltages, higher synaptic weights and unidirectional data flow capability. The present report is useful for the development and optimization of zinc oxide-based electronic synaptic devices for neuromorphic applications.

\section{Acknowledgements}

The authors thank Mr A. M. Teli for valuable discussion. This work was supported by funding from the School of Nanoscience and Biotechnology, Shivaji University, Kolhapur.

\section{REFERENCES}

1. Kuzum D, Yu S and Wong HP (2013) Synaptic electronics: materials, devices and applications. Nanotechnology 24(38): 3820011-382001-22.

2. Jo SH, Chang T, Ebong I et al. (2010) Nanoscale memristor device as synapse in neuromorphic systems. Nano Letters 10(4): 1297-1301.

3. Dongale TD, Khot KV, Mohite SV et al. (2016) Investigating the temperature effects on $\mathrm{ZnO}, \mathrm{TiO}_{2}, \mathrm{WO}_{3}$ and $\mathrm{HfO}_{2}$ based resistive random access memory (RRAM) devices. Journal of Nano- and Electronic Physics 8(4): 4030-1-4030-4.

4. Dongale TD, Patil KP, Gaikwad PK and Kamat RK (2015) Investigating conduction mechanism and frequency dependency of nanostructured memristor device. Materials Science in Semiconductor Processing 38: 228-233. 
Bioinspired, Biomimetic and Nanobiomaterials Volume 7 Issue BBN2
Effect of Ag doping on hydrothermally grown ZnO thin-film electronic synapse device

Mullani, Patil, Tikke et al.
5. Dongale TD, Patil KP, Mullani SB et al. (2015) Investigation of process parameter variation in the memristor based resistive random access memory (RRAM): effect of device size variations. Materials Science in Semiconductor Processing 35: 174-180.

6. Shinde SS and Dongle TD (2015) Modelling of nanostructured $\mathrm{TiO}_{2-}$ based memristors. Journal of Semiconductors 36(3): 0340011-034001-3.

7. Xia Q, Robinett W, Cumbie MW et al. (2009) Memristor-CMOS hybrid integrated circuits for reconfigurable logic. Nano Letters 9(10): 3640-3645.

8. Guan W, Long S, Liu Q, Liu M and Wang W (2008) Nonpolar nonvolatile resistive switching in $\mathrm{Cu}$ doped $\mathrm{ZrO}_{2}$. IEEE Electron Device Letters 29(5): 434-437.

9. Dongale TD, Khot KV, Mali SS et al. (2015) Development of Ag/ZnO/ FTO thin film memristor using aqueous chemical route. Materials Science in Semiconductor Processing 40: 523-526.

10. Dongale TD, Mohite SV, Bagade AA et al. (2015) Development of $\mathrm{Ag} / \mathrm{WO}_{3} / \mathrm{ITO}$ thin film memristor using spray pyrolysis method. Electronic Materials Letters 11(6): 944-948.

11. Dongale TD, Shinde SS, Kamat RK and Rajpure KY (2014) Nanostructured $\mathrm{TiO}_{2}$ thin film memristor using hydrothermal process. Journal of Alloys and Compounds 593: 267-270.

12. Xu N, Liu LF, Sun X et al. (2008) Bipolar switching behavior in TiN/ $\mathrm{ZnO} / \mathrm{Pt}$ resistive nonvolatile memory with fast switching and long retention. Semiconductor Science and Technology 23(7): 0750191-075019-4.

13. Dongale TD, Desai ND, Khot KV et al. (2016) Effect of surfactants on the data directionality and learning behaviour of $\mathrm{Al} / \mathrm{TiO}_{2} / \mathrm{FTO}$ thin film memristor-based electronic synapse. Journal of Solid State Electrochemistry 21(9): 2753-2757.

14. Dongale TD, Pawar PS, Tikke RS et al. (2018) Mimicking the synaptic weights and human forgetting curve using hydrothermally grown nanostructured $\mathrm{CuO}$ memristor device. Journal of Nanoscience and Nanotechnology 18(2): 1-8.

15. Waser R and Aono M (2007) Nanoionics-based resistive switching memories. Nature Materials 6(11): 833-840.

16. Nunes $P$, Fortunato $E$, Tonello $P$ et al. (2002) Effect of different dopant elements on the properties of $\mathrm{ZnO}$ thin films. Vacuum 64(3): 281-285.

17. Wang XL, Shao Q, Leung CW and Ruotolo A (2013) Non-volatile, reversible switching of the magnetic moment in Mn-doped $\mathrm{ZnO}$ films. Journal of Applied Physics 113(17): 17C301-1-17C301-3.

18. Younis A, Chu D and Li S (2013) Bi-stable resistive switching characteristics in Ti-doped $\mathrm{ZnO}$ thin films. Nanoscale Research Letters 8(1): 154-160.

19. Huang CY, Ho YT, Hung CJ and Tseng TY (2014) Compact Ga-doped $\mathrm{ZnO}$ nanorod thin film for making high-performance transparent resistive switching memory. IEEE Transactions on Electron Devices 61(10): 3435-3441.

20. Lee WH, Kim EJ and Yoon SM (2015) Effect of Al incorporation amount upon the resistive-switching characteristics for nonvolatile memory devices using Al-doped $\mathrm{ZnO}$ semiconductors. Journal of Vacuum Science \& Technology B, Nanotechnology and Microelectronics: Materials, Processing, Measurement, and Phenomena 33(5): 051216-1-051216-7.

21. Hosseini SM, Sarsari IA, Kameli P and Salamati H (2015) Effect of Ag doping on structural, optical, and photocatalytic properties of $\mathrm{ZnO}$ nanoparticles. Journal of Alloys and Compounds 640: 408-415.

22. Ahn BD, Kang HS, Kim JH et al. (2006) Synthesis and analysis of Agdoped ZnO. Journal of Applied Physics 100(9): 093701-1-093701-6.

23. Song YW, Kim K, Ahn JP, Jang GE and Lee SY (2009) Physically processed $\mathrm{Ag}$-doped $\mathrm{ZnO}$ nanowires for all- $\mathrm{ZnO} \mathrm{p}-\mathrm{n}$ diodes. Nanotechnology 20(27): 275606-1-275606-4.

24. Raza W, Faisal SM, Owais M, Bahnemann D and Muneer M (2016) Facile fabrication of highly efficient modified $\mathrm{ZnO}$ photocatalyst with enhanced photocatalytic, antibacterial and anticancer activity. RSC Advances 6(82): 78335-78350.

25. Shojaei AF, Tabatabaeian K, Zanjanchi MA, Moafi HF and Modirpanah N (2015) Synthesis, characterization and study of catalytic activity of Silver doped $\mathrm{ZnO}$ nanocomposite as an efficient catalyst for selective oxidation of benzyl alcohol. Journal of Chemical Sciences 127(3): 481-491.

26. Touam T, Boudjouan F, Chelouche A et al. (2015) Effect of silver doping on the structural, morphological, optical and electrical properties of sol-gel deposited nanostructured $\mathrm{ZnO}$ thin films. OptikInternational Journal for Light and Electron Optics 126(24): 5548-5552.

27. Jessell TM and Kandel ER (1993) Synaptic transmission: a bidirectional and self-modifiable form of cell-cell communication. Cell 72(7): 1-30.

28. Li S, Zeng F, Chen C et al. (2013) Synaptic plasticity and learning behaviours mimicked through Ag interface movement in an Ag/ conducting polymer/Ta memristive system. Journal of Materials Chemistry C 1(34): 5292-5298.

29. Chua L (1971) Memristor-the missing circuit element. IEEE Transactions on Circuit Theory 18(5): 507-519.

30. Simanjuntak FM, Panda D, Wei KH and Tseng TY (2016) Status and prospects of $\mathrm{ZnO}$-based resistive switching memory devices. Nanoscale Research Letters 11(1): 368-1-368-31.

31. Lin CY, Wu CY, Wu CY, Lin CC and Tseng TY (2007) Memory effect of RF sputtered $\mathrm{ZrO} 2$ thin films. Thin Solid Films 516(2): 444-448.

32. Kim KM, Jeong DS and Hwang CS (2011) Nanofilamentary resistive switching in binary oxide system: a review on the present status and outlook. Nanotechnology 22(25): 254002-1-254002-17.

\section{How can you contribute?}

To discuss this paper, please submit up to 500 words to the journal office at journals@ice.org.uk. Your contribution will be forwarded to the author(s) for a reply and, if considered appropriate by the editor-in-chief, it will be published as a discussion in a future issue of the journal.

ICE Science journals rely entirely on contributions from the field of materials science and engineering. Information about how to submit your paper online is available at www.icevirtuallibrary.com/page/authors, where you will also find detailed author guidelines. 